\title{
Zika virus infects cells lining the blood-retinal barrier and causes chorioretinal atrophy in mouse eyes
}

\author{
Pawan Kumar Singh, ${ }^{1,2}$ John-Michael Guest, ${ }^{1}$ Mamta Kanwar, ${ }^{1}$ Joseph Boss, ${ }^{1}$ Nan Gao, ${ }^{1}$ \\ Mark S. Juzych, ${ }^{1}$ Gary W. Abrams, ${ }^{1}$ Fu-Shin Yu, ${ }^{1,2}$ and Ashok Kumar, ${ }^{1,2,3}$ \\ 'Kresge Eye Institute, Department of Ophthalmology, ${ }^{2}$ Department of Anatomy and Cell Biology, and \\ ${ }^{3}$ Department of Microbiology, Immunology, and Biochemistry, Wayne State University School of Medicine, \\ Detroit, Michigan, USA.
}

\begin{abstract}
Zika virus (ZIKV) is an important pathogen that causes not only neurologic, but also ocular, abnormalities. Thus, it is imperative that models to study ZIKV pathogenesis in the eye are developed to identify potential targets for interventions. Here, we studied ZIKV interactions with human retinal cells and evaluated ZIKV's pathobiology in mouse eyes. We showed that cells lining the blood-retinal barrier (BRB), the retinal endothelium, and retinal pigment epithelium (RPE) were highly permissive and susceptible to ZIKV-induced cell death. Direct inoculation of ZIKV in eyes of adult C57BL/ 6 and IFN-stimulated gene 15 (ISG15) KO mice caused chorioretinal atrophy with RPE mottling, a common ocular manifestation of congenital ZIKV infection in humans. This response was associated with induced expression of multiple inflammatory and antiviral (IFNs) response genes in the infected mouse retina. Interestingly, ISC15 KO eyes exhibited severe chorioretinitis, which coincided with increased retinal cell death and higher ZIKV replication. Collectively, our study provides the first evidence to our knowledge that ZIKV causes retinal lesions and infects the cells lining the BRB and that ISG15 plays a role in retinal innate defense against ZIKV infection. Our mouse model can be used to study mechanisms underlying ZIKV-induced chorioretinitis and to gauge ocular antiviral therapies.
\end{abstract}

\section{Introduction}

The recent Zika virus (ZIKV) epidemic has posed significant challenges for health care and for the economy of Brazil and other affected countries $(1,2)$. While ZIKV infection during pregnancy has been linked primarily to microcephaly, a birth defect, the overall magnitude of risk remains uncertain (3-7). In view of limited data, microcephaly may represent only one possible adverse outcome among a spectrum of conditions of congenital Zika syndrome (4). Indeed, several clinical studies have reported ocular abnormalities, specifically in the retina and choroid, of infants with congenital ZIKV infection (8-13). Although currently undetermined, if a causal link between ZIKV infection and ocular disorders is established conclusively, it will have significant long-term economic, psychological, and health implications. Vision problems may be an underreported effect of the mosquito-transmitted ZIKV, which has spread in recent years from Africa and Asia to the Caribbean, Latin America, and parts of the United States (14). Thus, there is an urgent need to understand the pathogenesis of ZIKV in the eye and develop strategies to prevent potential vision loss due to ZIKV and other viral infections.

The involvement of the eye in ZIKV was first reported in 10 of 29 microcephalic Brazilian babies with

Conflict of interest: The authors have declared that no conflict of interest exists.

Submitted: December 14, 2016 Accepted: January 10, 2017 Published: February 23, 2017

Reference information: JCI Insight. 2017;2(4):e92340. https:// doi.org/10.1172/jici.nsight.92340. presumed congenital ZIKV infection whose eyes showed circumscribed variably sized areas of chorioretinal atrophy (11 eyes), circumscribed pigment mottling (11 eyes), optic nerve abnormalities (8 eyes), iris colobomas (2 eyes), and lens subluxation (1 eye) (9). Later, Miranda et al. described an extended spectrum of ocular manifestations of ZIKV in the eyes of 3 infants from northern Brazil born with microcephaly (8). Among these additional findings were hemorrhagic retinopathies, absence or early termination of retinal blood vessels, and torpedo maculopathy. As microcephaly from other causes (e.g., genetic) has also been reported to cause retinal abnormalities (15-17), the ocular findings described in these reports have been debated $(13,18)$. However, the ocular manifestations of ZIKV closely resemble those caused by other RNA 
viruses, such as chikungunya (19-21), dengue virus (DENV) (22-24), and West Nile virus (WNV) $(25,26)$, in adults, including optic neuritis, retinal hemorrhages, and retinal edema. Therefore, it is highly unlikely that the microcephaly itself causes retinal abnormalities. Indeed, a recent report shows chorioretinal scars in an infant without microcephaly (11). Given the heterogeneity of ocular manifestations of ZIKV, and the importance of defining the cause-and-effect relationship between ZIKV infection and ocular abnormalities, there is a need to develop animal models of this disease.

Recently, Miner et al. described the first animal model of ZIKV-induced eye disease, by subcutaneous injection of ZIKV in immunocompromised, type I IFN receptor $\mathrm{KO}$ (IFNRA1 ${ }^{-/}$) mice, and demonstrated panuveitis and shedding of viral RNA in tears of ZIKV-infected mice (27). This study also revealed the presence of ZIKV RNA in various ocular tissues, including the retina of IFNRA1 $1^{-1}$ mice, suggesting the entry of ZIKV into the eye. However, the mechanisms by which ZIKV gains access to the eye and causes inflammation remain to be determined. Similarly, the specific retinal cell(s) that support ZIKV replication in the eye and the immune response of human retinal cells to ZIKV challenge is currently unknown.

To fill in these knowledge gaps, we describe a model of direct inoculation of ZIKV in the eye to study its pathophysiology in causing ocular sequelae. Our data showed that ZIKV caused retinal lesions in immunocompetent (C57BL/6) and ISG15-deficient mouse eyes, with pigment clumping and retinal pigment epithelium (RPE) atrophy that mimicked ocular abnormalities described in humans (9). We also demonstrated that ZIKV infection caused cell death in primary human RPE and human retinal vascular endothelial cells (HRvEC), the cells lining the outer and inner blood-retinal barrier (BRB), respectively. Furthermore, our study is the first to our knowledge to report an important role of ISG15 in retinal innate immunity to ZIKV. Collectively, our in vitro and in vivo studies provide insights into the pathogenesis of ZIKV in the eye.

\section{Results}

ZIKV infects multiple retinal cell types and induces cell death. Eyes of infants with congenital ZIKV infection show pigmentary maculopathy, chorioretinal scarring, hemorrhage, and optic nerve and retinal blood vessel abnormalities (15-17), which suggest that the retina is the primary target of ZIKV in the eye. However, how ZIKV enters the eye and which retinal cells can support its replication are unknown. We hypothesized that cells lining the BRB are the first to encounter the blood-borne pathogens and tested ZIKV infectivity in vitro (Figure 1). ZIKV (strain PRVABC59, PR 2015) infected and replicated in both human primary RPE (Pr. RPE) cells and the ARPE-19 cell line, as evidenced by a time-dependent increase in the fluorescence intensity of viral staining in virus-infected cells (Figure 1B). Interestingly, Pr. RPE cells seemed to be more permissive to ZIKV infection than ARPE-19 cells, with overall higher infectivity. Similarly, ZIKV was found to infect primary HRvEC and Macaca primary choroidal endothelial cells (CEC), with HRvEC being more permissive to ZIKV replication (Figure 1). To determine whether ZIKV can infect other retinal cells, we tested the infectivity of human retinal Müller glia (MIO-M1 cell line) (28-30) and mouse cone photoreceptor cells (661W cell line) (31). While ZIKV-infected retinal Müller glia readily, photoreceptor cells seemed less permissive to ZIKV, as evidenced by fewer viral positive cells, even at later time points.

Because all retinal cells were found to be permissive to ZIKV to varying degrees, we next determined whether ZIKV induces cell death, a phenomenon commonly observed in viral infection. We used caspase-3 as a marker (32-34) and assessed its expression following ZIKV infection in the most permissive cell types, Pr. RPE cells and HRvEC. ZIKV significantly induced the expression of caspase-3 mRNA in a time-dependent manner, as assessed by quantitative PCR (qPCR) (Figure 2A). This finding was further confirmed by immunostaining of cleaved caspase-3 at the cellular level (Figure 2B). To ascertain ZIKV-induced cell death, the TUNEL assay was performed; the number of TUNEL-positive cells correlated with cleavage/activation of caspase-3 in RPE and HRvEC (Figure 2C). Similarly, ZIKV induced the cleavage of caspase-3 in the ARPE 19 cell line, retinal Müller glia, and CEC but not in the photoreceptor cells (Supplemental Figure 1; supplemental material available online with this article; doi:10.1172/jci.insight.92340DS1). Collectively, these results demonstrate that ZIKV can readily infect cells lining the BRB and induce cell death in the retina.

$Z I K V$ induces the expression of TAM receptors in retinal cells. Three homologous type I receptor tyrosine kinases - TYRO3, AXL, and MERTK (TAMs) - have been implicated in ZIKV entry in various cell types, including fibroblasts, keratinocytes, dendritic cells, placental cells, and neuronal cells (35-37). As the first step to determine the potential role of TAM receptors in ZIKV invasion in the eye, we assessed their expression in human Pr. RPE cells and HRvEC. Compared with the mock control, ZIKV challenge 
A
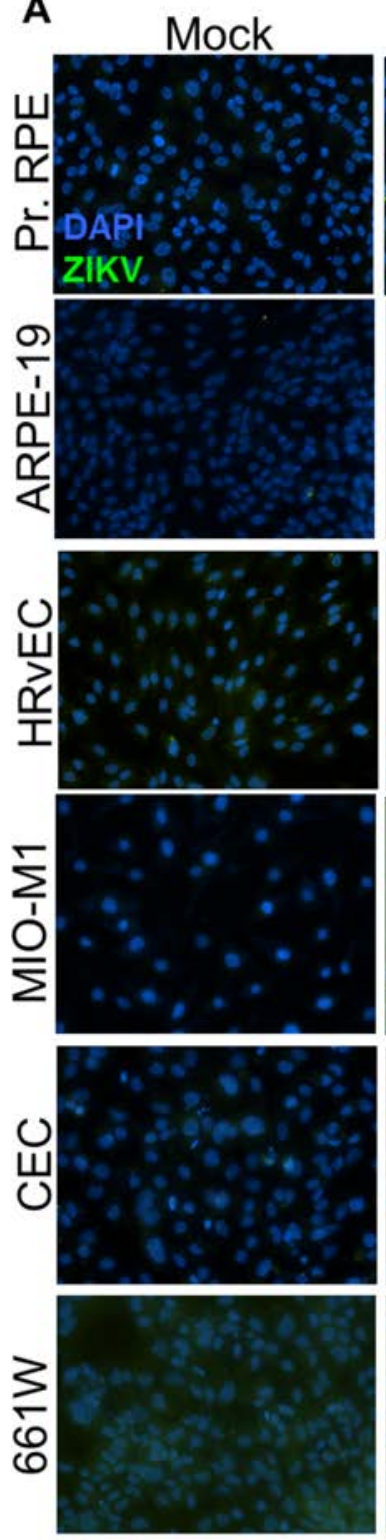

$24 \mathrm{hpi}$
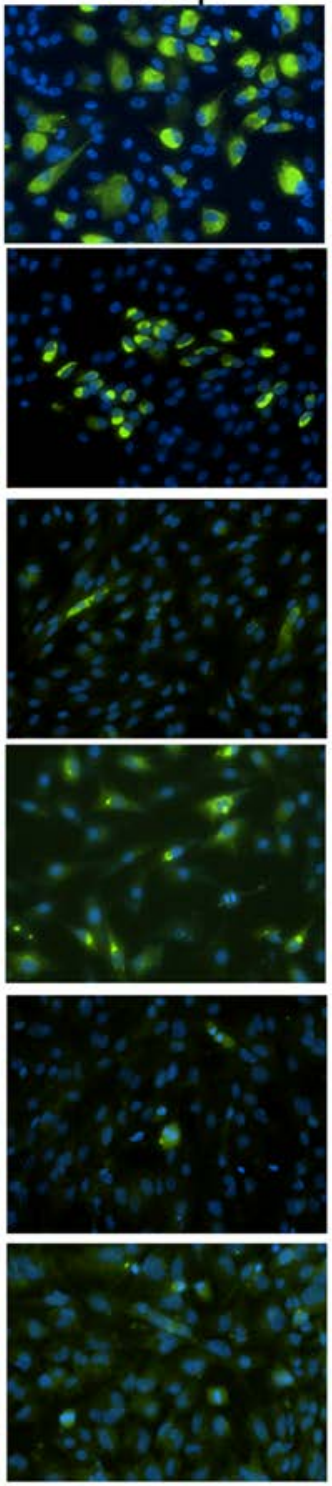
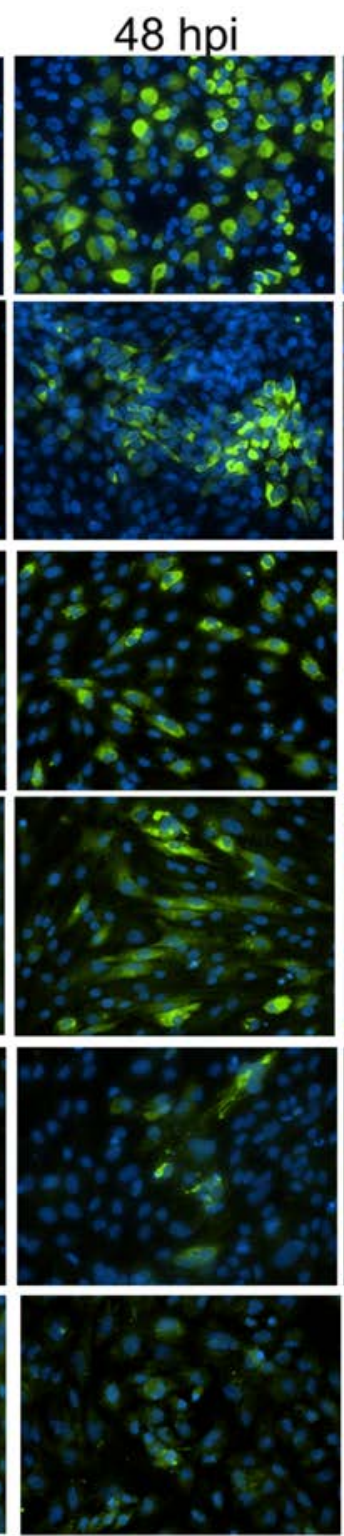

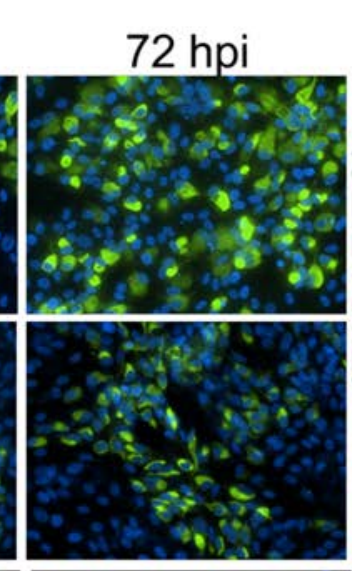

B
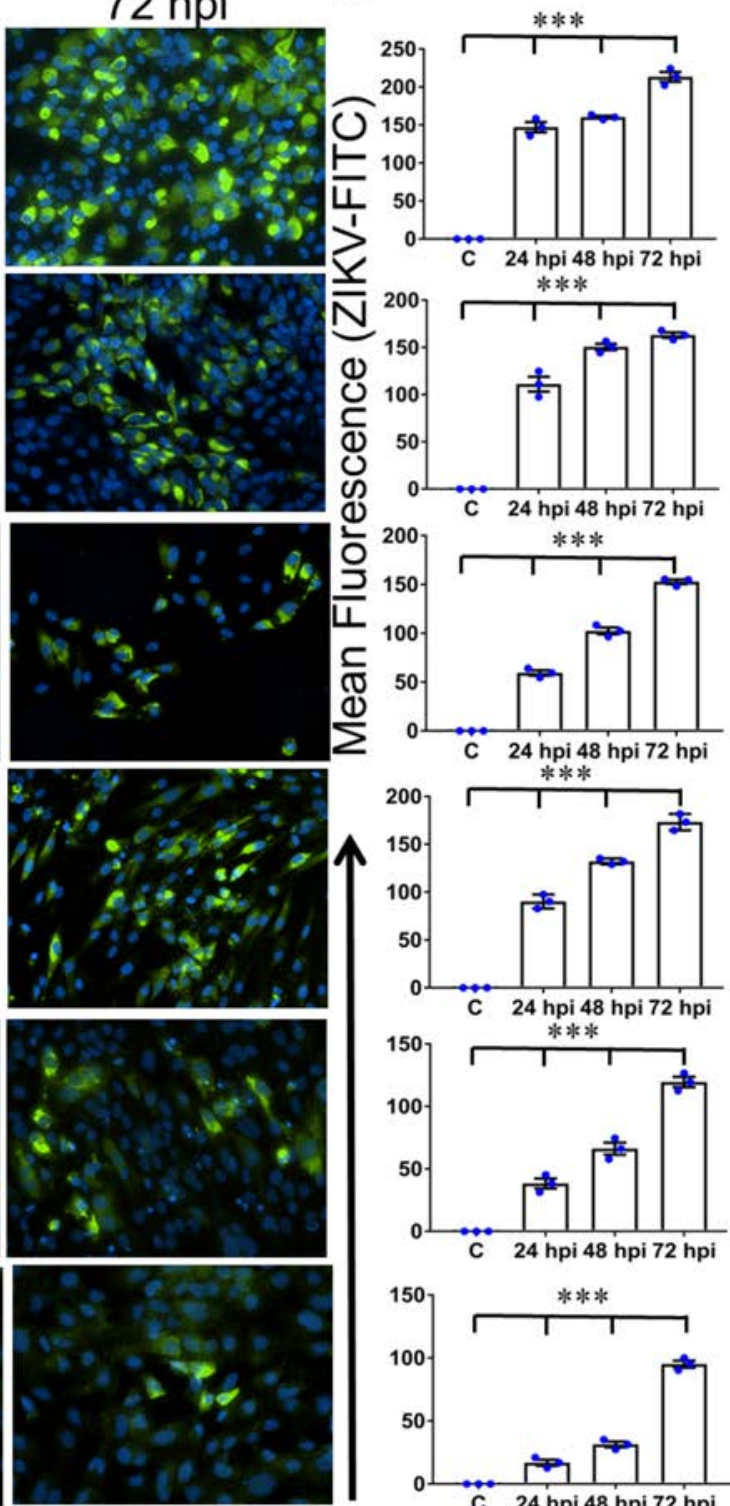
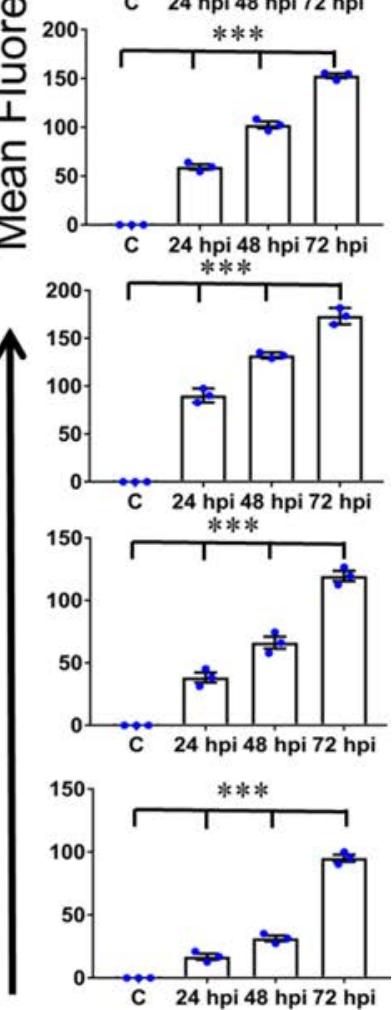

Figure 1. ZIKV infects multiple human retinal cell types. (A) Human primary retinal pigment epithelial cells (Pr. RPE), the immortal RPE cell line (ARPE-19), primary retinal vascular endothelial cells (HRvEC), the immortal retinal Müller glia cell line (MIO-M1), choroidal endothelial cells (CEC), and the retinal cone photoreceptor cell line (661W) were challenged with ZIKV (strain PRVABC59, PR 2015, MOI of 1) for the indicated time points; uninfected cells served as mock control. Control and ZIKV-infected cells were subjected to immunostaining for antiflavivirus group antigen 4G2, and representative images show the presence of ZIKV (green) and DAPI (blue, a cell nuclear stain). hpi, hours postinfection. Original magnification, $\times 20$. (B) Image) quantification of the mean fluorescence intensity (MFI) for viral staining (mean $\pm \mathrm{SEM} ; n=3$; *** $P<0.0001 ; 1$-way ANOVA).

significantly upregulated the expression of TAM receptors at the mRNA level in a time-dependent manner (Figure 3A). Since AXL was identified as the main candidate receptor for ZIKV, its expression was confirmed at the cellular level by immunostaining (Figure 3B) and at the protein level by Western blot (Figure 3C) analyses. Similar to RPE and HRvEC, ZIKV infection induced the expression of AXL in all retinal cell types except photoreceptor cells (Supplemental Figure 2). To further investigate the role of AXL as an entry/attachment factor to retinal cells, we used a selective inhibitor of AXL R428, and our results show that inhibition of AXL receptor completely blocked ZIKV infection to the Pr. RPE cells (Figure 3D). These findings suggest that ZIKV may use TAM receptors for entry in certain retinal cells.

$Z I K V$ elicits antiviral innate response in retinal cells. As ZIKV was found to infect multiple retinal cell types and to induce the expression of multiple potential entry receptors, we next determined whether ZIKVinfected retinal cells elicit an innate immune response. TLRs have been shown to play an important role 


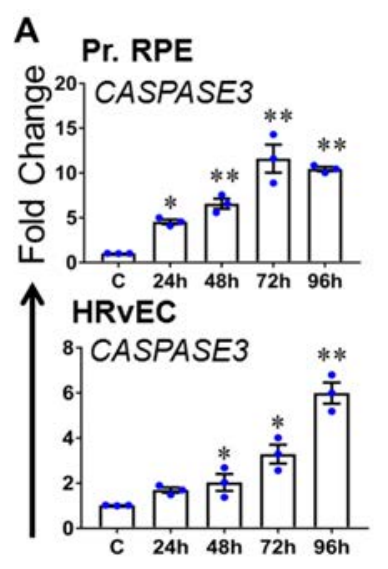

B
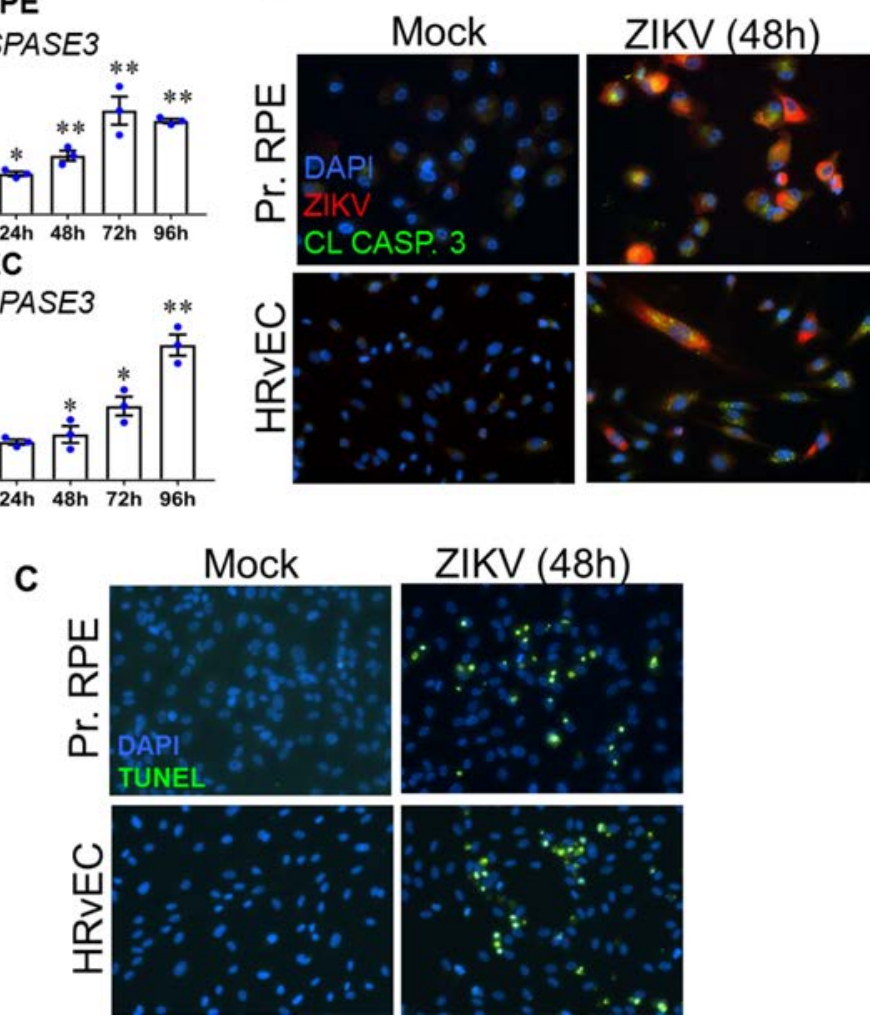

Figure 2. ZIKV infection causes retinal cell death. (A) qPCR showing upregulation of caspase-3 transcripts in primary retinal pigment epithelial (Pr. RPE) cells and human retinal vascular endothelial cells (HRvEC) following ZIKV infection (strain PRVABC59, PR 2015, MOI of 1) (mean \pm SEM; $n=3$; ${ }^{*} P<0.05$, ${ }^{*} P<0.005$; Student's $t$ test). (B) Representative immunofluorescence images showing immunostaining for cleaved caspase-3 (CL Casp.3, green), ZIKV (red), and DAPI (blue) in Pr. RPE cells and HRvEC 48 hours following ZIKV infection and mock treatment. See also Supplemental Figure 1. (C) Representative images showing TUNEL-positive cells (green) and DAPI (blue) in Pr. RPE cells and HRvEC 48 hours after ZIKV infection and mock treatment. Original magnification, $\times 20$.

followed by a time-dependent decline while TLR3 levels remained elevated.

To determine the biological relevance of ZIKV-induced innate immune receptor expression, we assessed the expression of proinflammatory mediators, IFNs, and IFN-stimulated antiviral genes. The qPCR analyses revealed increased mRNA transcripts of proinflammatory cytokines TNFA, IL1B, and IL6; chemokines CCL5 and CXCL10; and IFNs IFNA2, IFNB1, and IFNG in ZIKV-infected Pr. RPE cells (Figure 4A) and HRvEC (Figure 5A); but these transcripts varied in their expression pattern. Pr. RPE cells showed a time-dependent increase in the levels of TNFA and IL6, whereas IL1B peaked at 24 hours followed by a gradual decline. In contrast, in HRvEC, TNFA and IL6 peaked at 72 and 48 hours, respectively, and then decreased with time, while $I L 1 B$ showed a time-dependent increase. The expression time course of IFNs and antiviral chemokines CCL5 and CXCL10 also varied between Pr. RPE cells and HRvEC. Coinciding with IFN expression, we observed significant upregulation of IFN-stimulated antiviral response genes, specifically, ISG15, OAS2, and $M X 1$, in ZIKV-infected Pr. RPE cells and HRvEC. The expression of ISG15 at the protein level was confirmed by immunostaining (Figure $4 \mathrm{C}$ and Figure $5 \mathrm{C}$ ). These results indicate that human retinal cells possess the ability to respond to ZIKV and elicit an inflammatory and antiviral response.

Ocular inoculation of ZIKV caused chorioretinal atrophy in adult mouse eyes. Having shown that ZIKV infects retinal cells, induces an inflammatory response, and causes cell death, we sought to determine whether ZIKV causes ocular pathology similar to that seen in human patients. First, ZIKV was administrated subcutaneously in adult B6 mice ( $10^{5} \mathrm{PFU} /$ mouse) followed by a daily eye exam for up to 7 days; we did not observe ocular pathology in the eyes of adult B6 mice (data not shown). Next, we tested whether direct inoculation of ZIKV causes ocular pathology and followed the consequences of ZIKV infection of the eye over time. While uninfected control eyes had clear media, flat optic discs, normal blood vessels, and clear maculae without foveal reflexes, ZIKV-injected eyes developed white subretinal lesions with soft edges radiating from the optic discs within 24 hours. At 48 hours after ZIKV infection, the media became slightly hazy, the optic discs and rims became less apparent, and large white subretinal lesions with early stages of chorioretinal atrophy with poorly defined edges appeared. As the disease progressed at 72 and 96 hours, the white subretinal lesions exhibited RPE atrophy and pigment mottling within large fibrotic chorioretinal scars (Figure 6A).

Due to ZIKV-induced expression of ISG15 in Pr. RPE cells and HRvEC (Figures 4 and 5), and the 
A
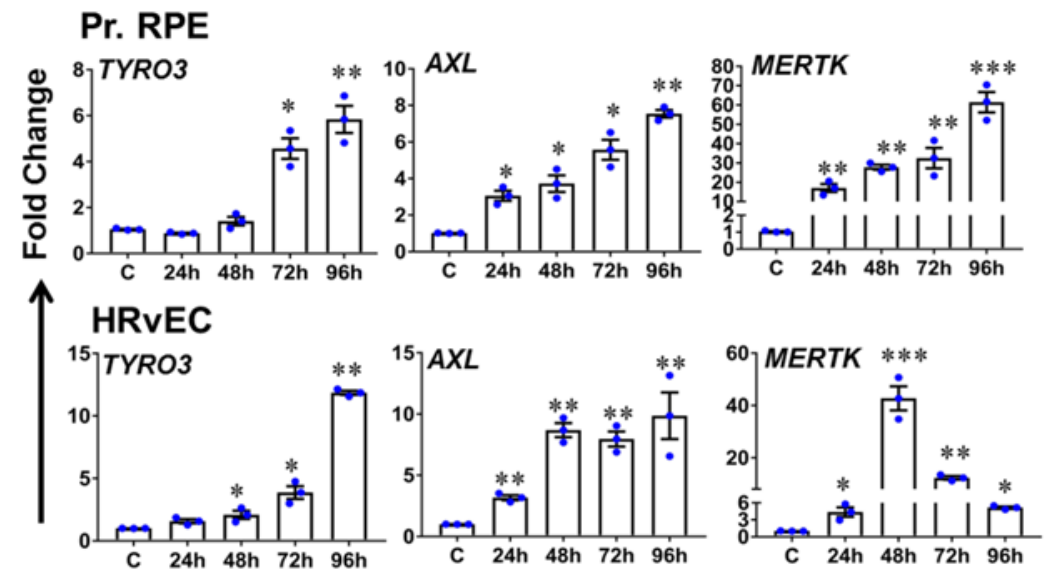

B
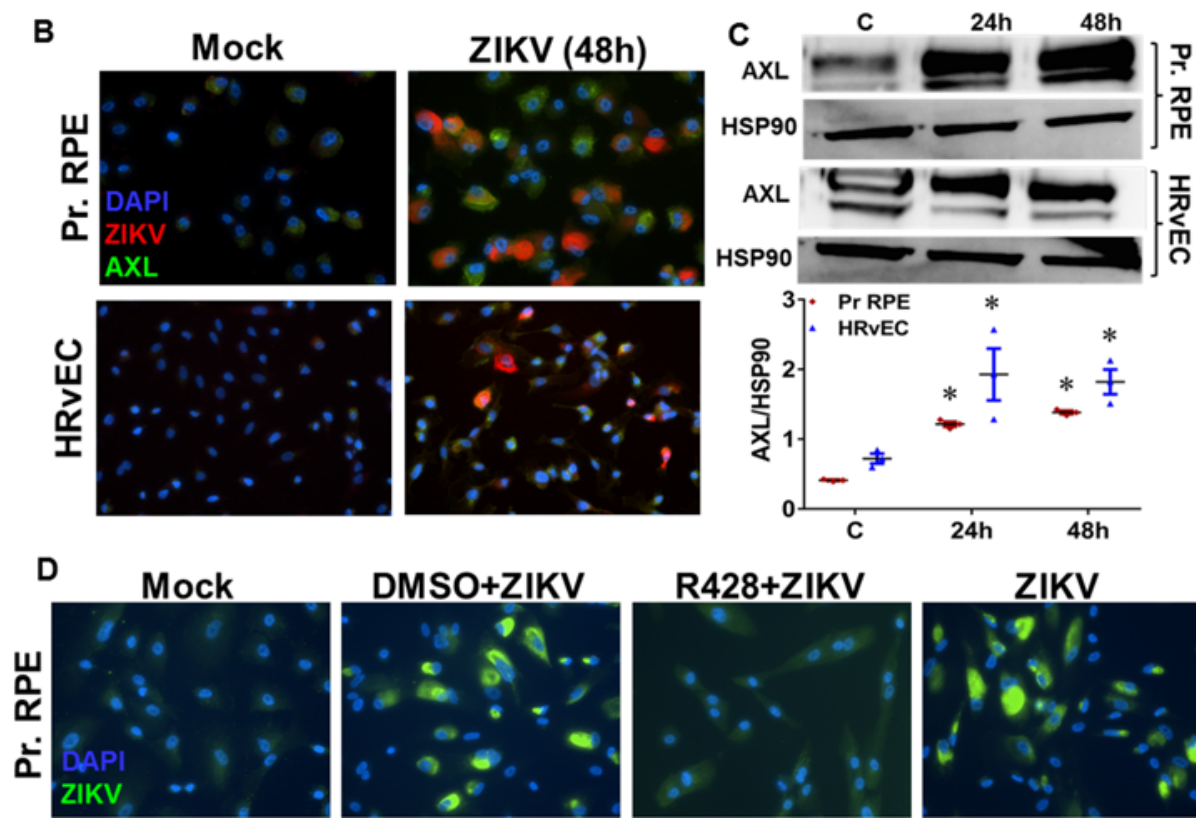

Figure 3. ZIKV induces TAM receptor expression in Pr. RPE cells and HRvEC. (A) qRT PCR showing upregulation of TAM receptors TYRO3, AXL, and MERTK in primary retinal pigment epithelial (Pr. RPE) cells and human retinal vascular endothelial cells (HRVEC) following ZIKV infection (strain PRVABC59, PR 2015, MOI of 1) (mean \pm SEM; $n=3$; ${ }^{*} P<$ $0.05,{ }^{* *} P<0.005,{ }^{* * *} P<0.0005$; Student's $t$ test). (B) Representative immunofluorescence images showing immunostaining for AXL (green), ZIKV (red), and DAPI (blue) at 48 hours after ZIKV infection in Pr. RPE cells and HRvEC. (C) Western blot showing expression of AXL in Pr. RPE cell and HRvEC lysates 24 and 48 hours after ZIKV infection. The bar graph represents densitometry analysis of Western blots using ImageJ with respect to HSP9O as housekeeping control (mean $\pm \mathrm{SD}$; ${ }^{*} P<0.05$; Student's $t$ test). See also Supplemental Figure 2. (D) Representative immunofluorescence images showing immunostaining for ZIKV (green) and DAPI (blue) following AXL inhibition using R428 (1 $\mu \mathrm{M}$ for 2 hours) followed by ZIKV infection for 48 hours in Pr. RPE cells. (B and D) Original magnification, $\times 20$.

well-established antiviral role of ISG15 $(42,43)$, we postulated that ISG15 might play a role in retinal defense against ZIKV. To test this, we used ISG $15^{-/-}$mice and assessed ocular pathology following intravitreal ZIKV infection. The timecourse study revealed that, similar to WT mice, retinal lesions in $\mathrm{ISG} 15^{-/}$mice were visible within 24 hours of ZIKV infection. However, in comparison with

WT mice, the eyes of ZIKV-infected ISG15 ${ }^{-/-}$mice showed severe chorioretinitis, characterized by RPE atrophy, clumping, and mottling, with marbleization at later time points, i.e., 72 and 96 hours after infection (Figure 6, A and B). The increased severity of retinal lesions in ISG15 $5^{-1-}$ mice coincided with higher levels of viral RNA (Figure 6C) and viral proteins (Supplemental Figure 3) in ISG15 ${ }^{-/}$retinas. Histological analysis of the ZIKV-infected retinas revealed an increased retinal cell death in ISG15 ${ }^{-/-}$mice, with a higher number of TUNEL-positive (+ve) cells (Figure 6D). Together, our data demonstrate that ZIKV causes retinal lesions in adult mouse eyes, and ISG15 deficiency increases disease severity.

ISG15 deficiency diminishes the retinal antiviral response to ZIKV infection. Next, we determined whether ZIKV evokes innate immune responses in the eye. Similar to our in vitro studies (Figures 4 and 5), ZIKV induced the time-dependent upregulation of Tlr3 and RigI receptor mRNA expression in WT mice as compared with ISG15 ${ }^{-/-}$mice (Figure 7), whereas Mda5 receptor mRNA expression was similar. Among the TAM receptors, the expression of $A x l$ and Mertk was significantly higher in WT retinas versus ISG $15^{-/-}$retinas at all time points. We found significantly higher transcripts of Il6 in WT mice as compared with ISG15 ${ }^{-/-}$mice, while Tnfa and $I l 1 b$ transcript levels were comparable (Figure 7). Analysis of the IFN response showed higher levels of Ifn $1 b$ at the 48-hour time point and of Ifng at the 24-hour time point in WT mice as compared with ISG15/- mice, indicating an early IFN response in WT mice. At the later time points, the IFN transcripts were comparable in both animals. Similarly, the levels of antiviral chemokines ( $C x c 110$ and $C c 15)$ and IFN-induced anti-viral genes (Isg15, Oas2, and Mx1) showed a time-dependent increase in WT mice versus ISG15 ${ }^{-1-}$ mice. Collectively, these results indicate the existence of a strong antiviral immune response in WT mice versus ISG15-deficient mice toward ZIKV infection in the eye. 
A
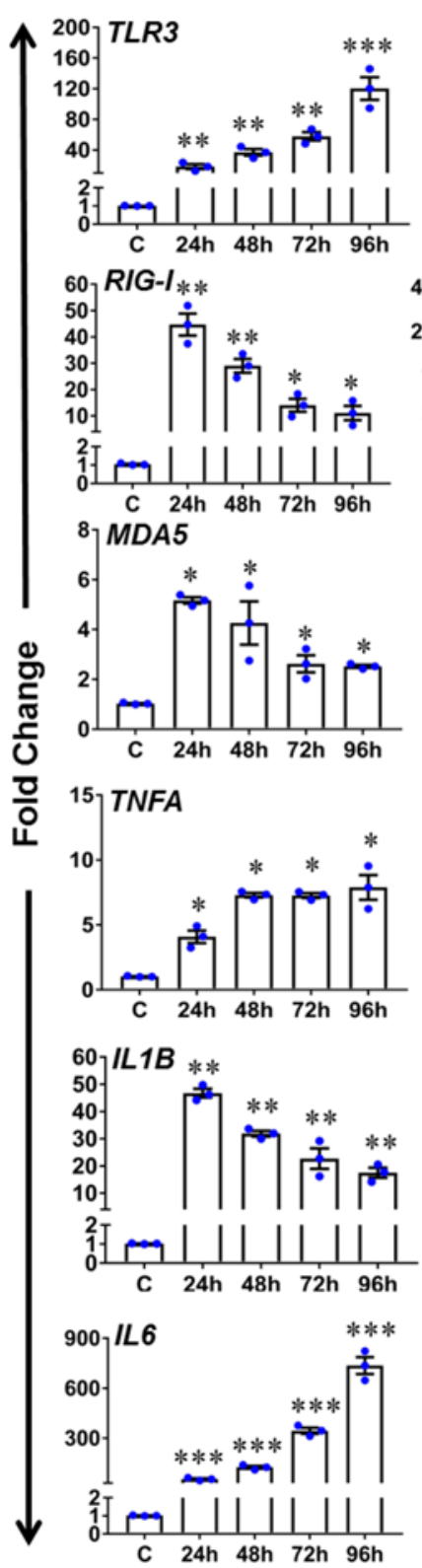
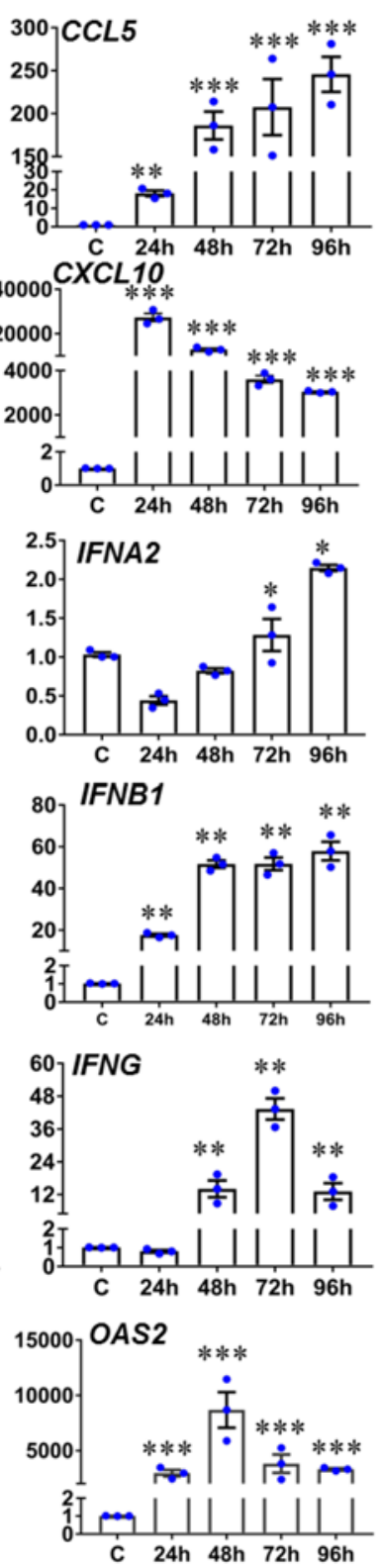
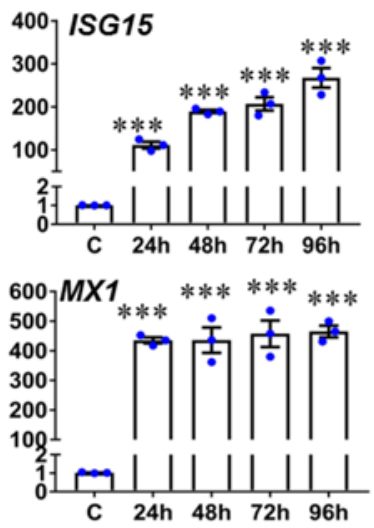

B
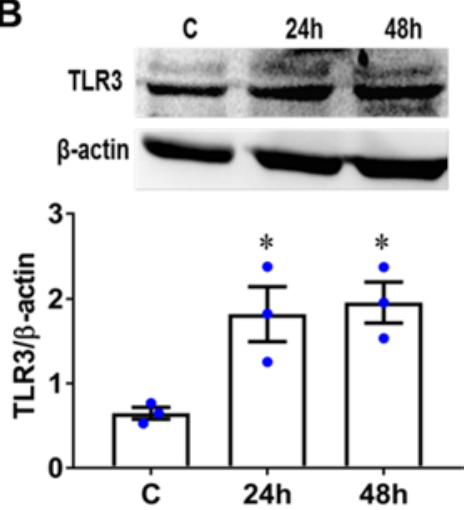

C
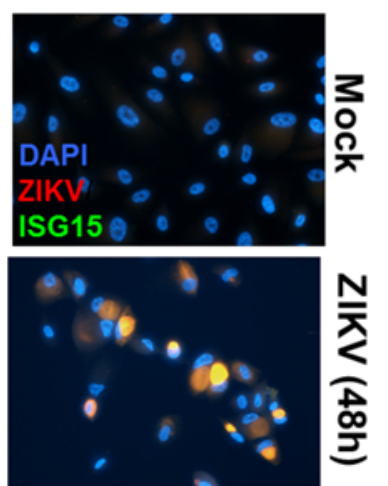

Figure 4. ZIKV evokes innate inflammatory and antiviral responses in Pr. RPE cells. (A) qRT PCR showing mRNA expression of PRRs (TLR3, RIGI, and $M D A 5$ ), inflammatory mediators (TNFA, IL1B, IL6, CCL5, and CXCL10), IFNs (IFNA2, IFNB1, and IFNG), and IFN-induced genes (OAS2, ISG15, and $M X 1)$ following ZIKV infection (strain PRVABC59, PR 2015, MOI of 1) (mean \pm SEM; $n=3 ;{ }^{*} P<0.05,{ }^{* *} P<0.005$, ${ }^{* * *} P<0.0005$, Student's $t$ test). (B) Western blot showing expression of TLR3 in primary retinal pigment epithelial (Pr. RPE) cell lysates 24 and 48 hours after ZIKV infection. The bar graphs represent densitometry analysis of Western blots using Image] with respect to $\beta$-actin housekeeping control (mean $\pm \mathrm{SEM} ; n=3$; ${ }^{*} P<0.05$; Student's $t$ test). (C) Representative immunofluorescence images showing staining for ISC15 (green), ZIKV (red), and DAPI (blue) 48 hours after ZIKV infection and mock treatment. Original magnification, $\times 20$.

\section{Discussion}

The emergence of ZIKV in both endemic and nonendemic regions of the world has been accompanied by an unprecedented rise in the spectra of ZIKV-associated diseases. It is becoming increasingly clear that ZIKV infection has broad implications beyond microcephaly, as infants born with congenital ZIKV have pathology of their eyes, ears, limbs, and perhaps other organs (44). Consequently, there has been significant interest in understanding the pathogenesis of ZIKV in nonneurological dis-

eases. Because clinical studies have linked ZIKV to ocular abnormalities, primarily in the retina of infants $(8,12)$ and adults $(45,46)$ and uveitis in adults $(45)$, it is important to study ZIKV pathogenesis in the eye to identify potential targets for intervention. We have shown that direct inoculation of ZIKV in the adult mouse eye results in retinal lesions with a clinical presentation (chorioretinal atrophy and RPE mottling) resembling some of the features of ZIKV-associated ocular pathology described in humans. We also observed that ZIKV infects multiple retinal cell types and incites an antiviral innate response in the retina. Most importantly, ISG15 was discovered as a key player in restricting viral replication in the retina/eye. Thus, our study reveals the cellular basis of ZIKV infectivity in the eye and provides experimental models to gauge antiviral or other immunotherapies to ameliorate potential vision loss due to ZIKV infection.

The major protective mechanism that controls the influx of innate immune cells and pathogens into the posterior segment of the eye is the presence of $\mathrm{BRB}$, consisting of the inner retinal vascular endothelial cells and outer RPE cells $(40,41,47)$. Previous studies from our laboratory and others have shown that BRB dysfunction allows the entry of infectious agents into the retina/vitreous cavity and causes inflammation and tissue damage $(48,49)$. A recent study by Miner, Apte, and colleagues provided the 
A
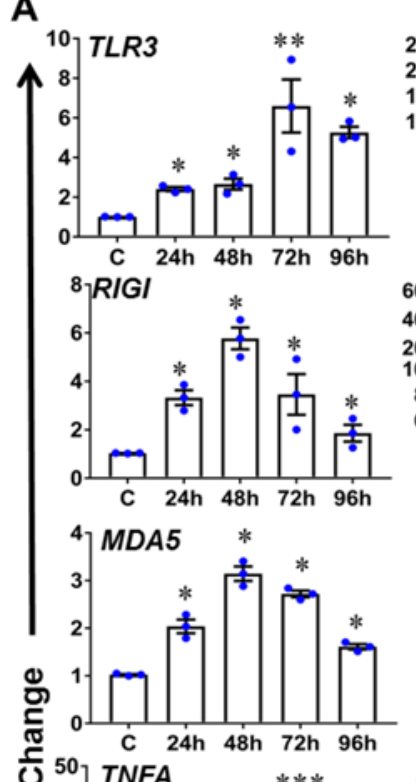
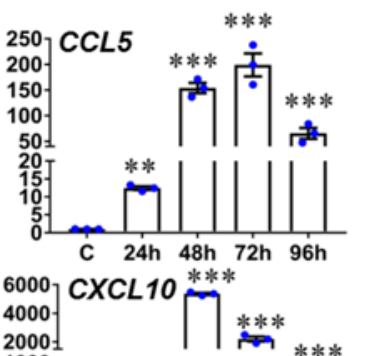

20001 舟必

800- $600-10 *||||||$

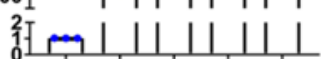

C $24 \mathrm{~h} 48 \mathrm{~h} \quad 72 \mathrm{~h} 96 \mathrm{~h}$

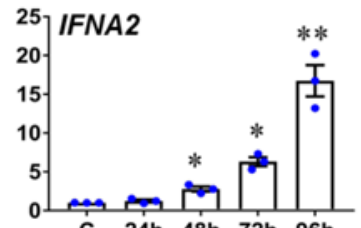

C $24 \mathrm{~h} 48 \mathrm{~h} 72 \mathrm{~h} 96 \mathrm{~h}$

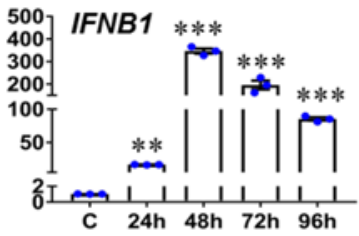

C $24 \mathrm{~h} \quad 48 \mathrm{~h} \quad 72 \mathrm{~h} \quad 96 \mathrm{~h}$
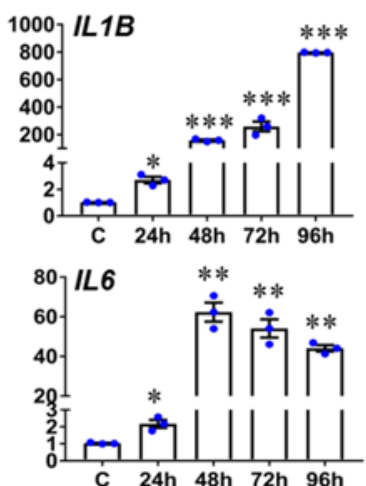

$24 \mathrm{~h} 48 \mathrm{~h} 72 \mathrm{~h} 96 \mathrm{~h}$

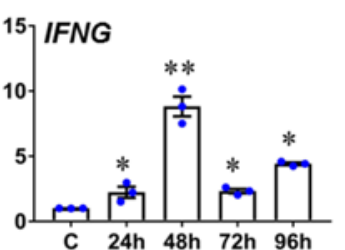

30_OAS2 $*$ * * *

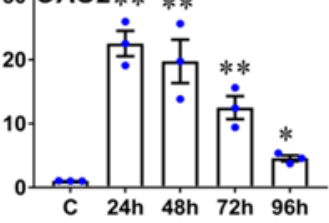

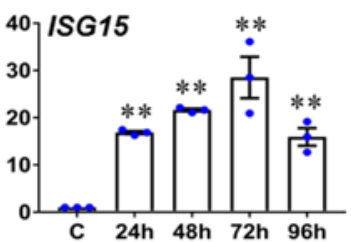

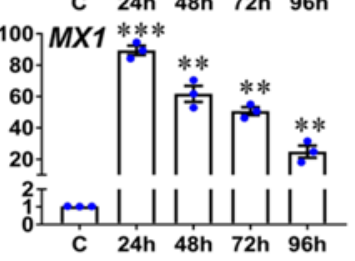

B
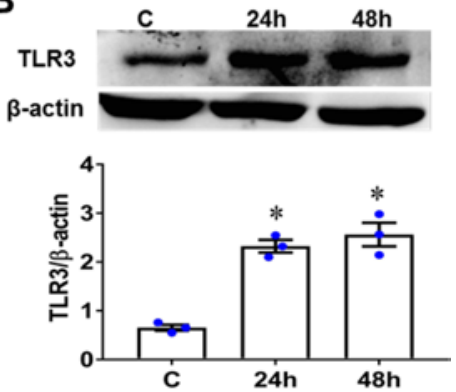

C

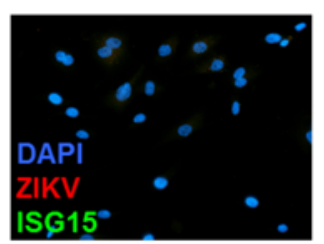

3
읏

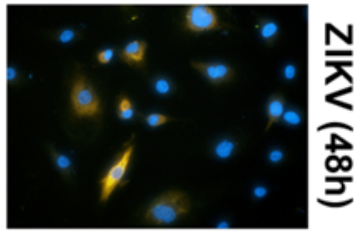

Figure 5. ZIKV evokes innate inflammatory and antiviral responses in HRvEC. (A) qRT PCR showing mRNA expression of PRRs (TLR3, RIGI, and MDA5), inflammatory mediators (TNFA, IL1B, IL6, CCL5, and CXCL10), IFNs (IFNA2, IFNB1, and IFNG), and IFN-induced antiviral genes (OAS2, ISG15, and MX1) following ZIKV infection (strain PRVABC59, PR 2015, MOI of 1) (mean $\pm \mathrm{SEM} ; n=3 ;{ }^{*} P<0.05,{ }^{* *} P<0.005,{ }^{* * *} P<$ 0.0005 ; Student's $t$ test). (B) Western blot showing the expression of TLR3 in human retinal vascular endothelial cell (HRvEC) lysate 24 and 48 hours after ZIKV infection. The bar graph represents densitometry analysis of Western blots using Image] with respect to $\beta$-actin housekeeping control (mean \pm SEM; $n=3$; ${ }^{*} P<0.05$; Student's $t$ test).(C) Representative immunofluorescence images showing staining for ISG15 (green), ZIKV (red), and DAPI (blue) 48 hours after ZIKV infection and mock treatment. Original magnification, $\times 20$.

first evidence of ZIKV RNA in the eyes of subcutaneously challenged IFNRA1 $^{-/-}$mice (27), but the cellular mechanisms by which ZIKV invaded the eye were not elucidated. We hypothesized that, being a blood-borne pathogen, ZIKV must overcome the BRB to cause retinal lesions, the key manifestation of ZIKV infection in the eye.

Because RPE and HRvEC constitute the outer and inner BRB, respectively, we evaluated the effects of ZIKV infection on these cell types and demonstrated that these cells are permissive to ZIKV replication.

Moreover, our data showed that ZIKV-infected RPE and HRvEC exhibited caspase-3 activation, coinciding with increased TUNEL positivity, evidence of cell death. Time-course studies revealed that Pr. RPE cells and HRvEC are more susceptible to ZIKV. These observations are significant on several levels: (a) RPE and retinal endothelial cells are likely among the principal retinal cell types infected by ZIKV, (b) ZIKV may gain access to the eye by directly infecting the cells lining the BRB, and (c) ZIKV-infected BRB cells may serve as a reservoir of ZIKV and facilitate viral dissemination to other organs, including the brain.

TAM receptors have been shown to facilitate the attachment and entry of several viruses (50), including the members of flaviviridae such as WNV (51), DENV (52), and ZIKV (53). To investigate the role of TAMs in ZIKV infection of the eye, we assessed the expression of TAMs - TYRO3, MERTK, and AXL - in retinal cells, specifically Pr. RPE cells and HRvEC. Our data showed that the expression of TAM receptors is significantly increased in cultured retinal cells following ZIKV infection. Similar to in vitro studies, ZIKV induced the expression of TAM receptors in the mouse retina, implying their involvement in ZIKV infection of the retina. Although deficiency of AXL and MERTK receptors has been shown to increase susceptibility to WNV infection (51), Miner et al. demonstrated that AXL and MERTK deficiency does not affect ZIKV replication in the eye, suggesting that these receptors are not required for CNS or ocular infection in mice (27). However, they did not completely rule out the 
A
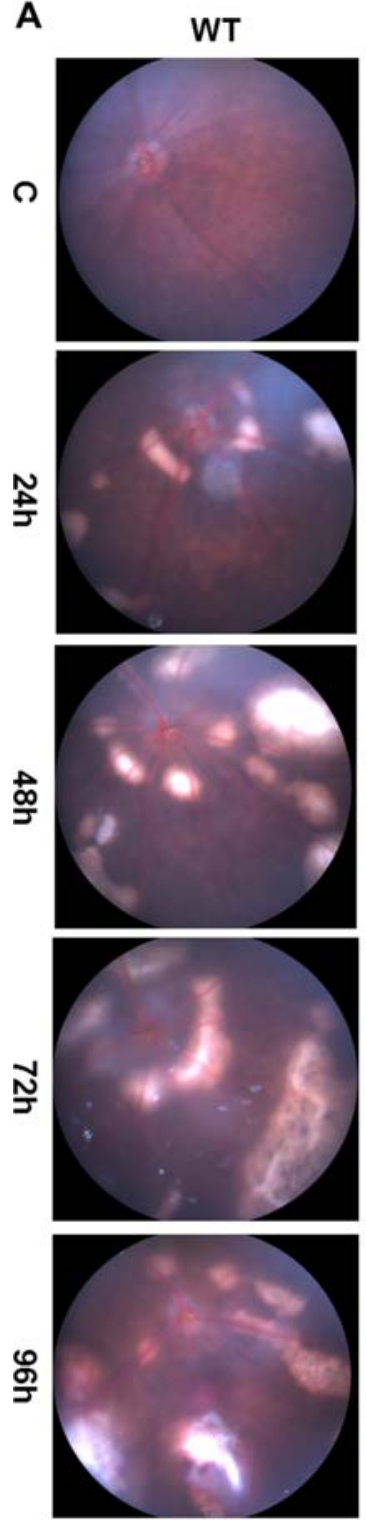
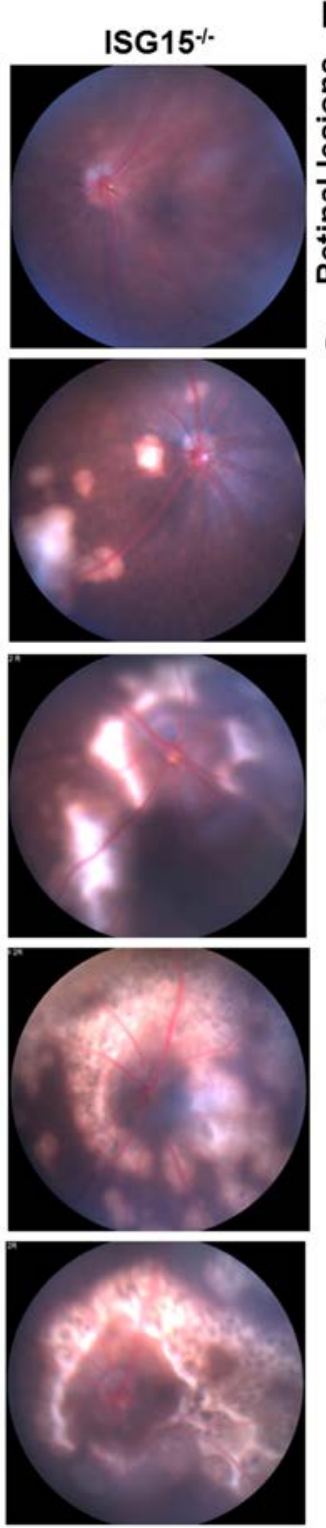
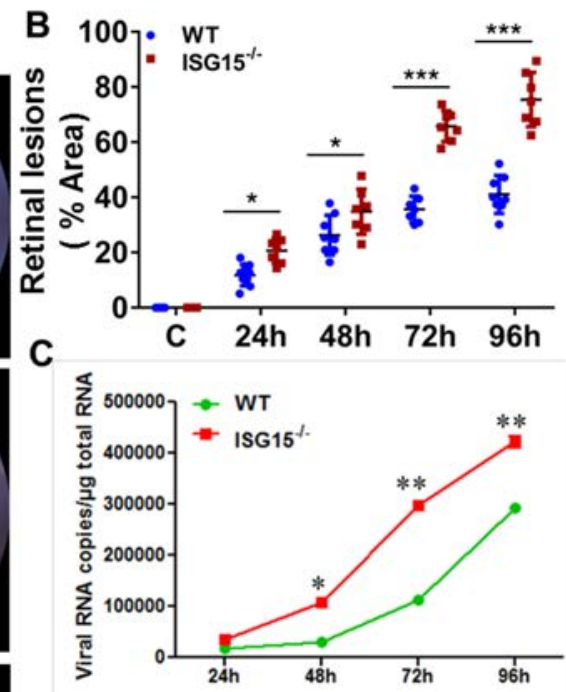

D
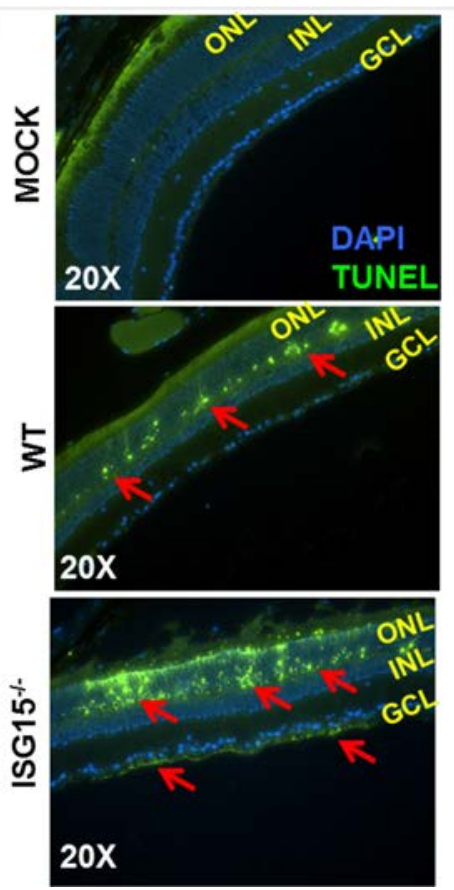

Figure 6. ZIKV causes retinal lesions in mouse eyes with chorioretinitis, RPE mottling, and cell death. Eyes of 4- to 6-week-old C57BL/6 and ISC15 1 - mice ( $n=$ 7-10/group) were injected intravitreally with $10^{4}$ PFU of ZIKV (strain PRVABC59, PR2015).

(A) Representative funduscopic images showing the time course of retinal lesion/ chorioretinal atrophy and RPE mottling in WT and ISC15 $5^{-1-}$ mice. (B) Quantification of the percentage area with retinal lesions per eye in WT and ISG15 $15^{-/}$mice at indicated time points $(24,48,72$, and 96 hours) using Image) analysis (mean $\pm \mathrm{SD} ;{ }^{*} P<0.05$, ${ }^{* *} P$ $<0.0005$; Student's $t$ test). (C) qRT PCR showing intracellular viral RNA quantification in WT and ISG15 1 - mouse retinas at various time points (mean $\pm \mathrm{SD}$; ${ }^{*} P<0.05$, ${ }^{*} P<0.005$; Student's $t$ test). (D) Representative images showing TUNEL-positive cells (green, indicated by the red arrows) and DAPI (blue) in various layers of the retinas of ZIKV-infected eyes (96 hours) and mock treatment. ONL, outer nuclear layer; INL, inner nuclear layer; GCL, ganglion cell layer. Original magnification, $\times 20$.

possibility that AXL is required as an entry factor for ZIKV in specific retinal cell types. In contrast, our study showed that inhibition of AXL signaling using a selective AXL inhibitor, R428, markedly blocked ZIKV replication in Pr. RPE cells. This finding also corroborated the study showing that AXL inhibition impaired ZIKV infection in the human blood-brain barrier endothelial cell line, hCMEC/D3 (54). These findings suggest that ZIKV may use AXL as an entry receptor to gain access to BRB cells and cause pathology in the retina.

Upon viral entry, host cells employ intracellular pathogen-recognizing receptors (PRRs), such as TLRs and RIG-I-like receptors, for recognition and initiation of innate immune responses, particularly, the generation of type I IFNs $(36,55)$. Our data show that ZIKV induced the expression of TLR3, RIGI, and MDA5 in Pr. RPE cells and HRvEC as well as in the retina, suggesting that these PRRs might be involved in recognition of ZIKV in the eye. PRR activation leads to the production of various inflammatory mediators, including cytokines, chemokines, and IFNs. The type I and type II IFNs have been shown to restrict flavivirus propagation, including DENV, WNV, and ZIKV $(56,57)$. Our results corroborate enhanced TNFA, IL6, $I L 1 B, C X C L 10, C C L 5, I F N A, I F N B$, and IFNG gene expression following ZIKV challenges both in vitro and in vivo. Moreover, ZIKV infection induced the expression of several IFN-induced antiviral genes, including ISG15, OAS2, and MX1. These IFN-stimulated genes (ISGs) have been shown to exert strong antiviral effects and restrict the viral replication of flaviviruses like DENV and WNV $(58,59)$. Marked upregulation of these ISGs suggests a role for type I IFNs in controlling ZIKV infection and replication in vivo.

To date, whether ZIKV can cause ocular pathology in animal models, similar to those reported in human patients, is not known $(8,9,45,60)$. Thus, the availability of suitable animal models that can define the tissue pathology associated with the disease manifestations may provide better insight into the pathogenesis and potentially future prevention and treatment of ZIKV infections. In this study, we demonstrate that direct inocu- 


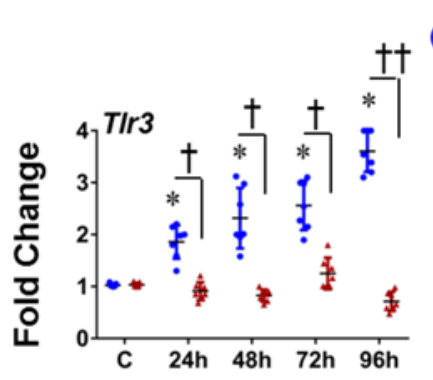

WT $\triangle$ ISG15 $^{-1-}$
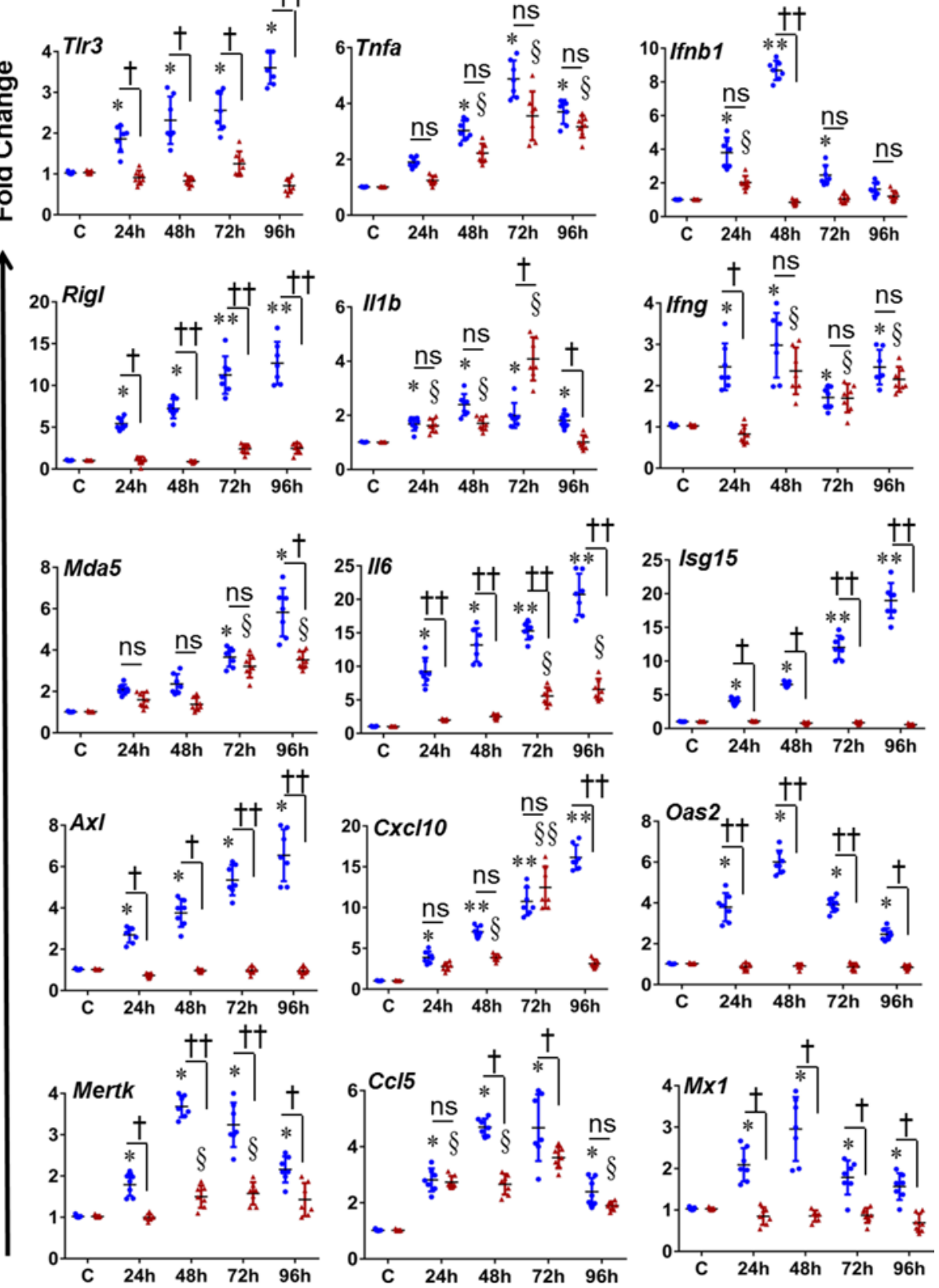

lation of ZIKV resulted in development of retinal lesions in adult WT mice, with evidence of choroidal inflammation and RPE damage/atrophy. Our intravitreal inoculation method bypasses the BRB and infects the retina directly. We acknowledge the limitations of our animal model, in particular, in deciphering if and how ZIKV crosses the BRB. However, the observed ocular pathology in our model that closely resembles ZIKV-induced retinal lesions in humans provides advantages over IFN$\mathrm{RA}^{-/-}$mice (27) for studying the mechanisms of pathogenesis of chorioretinal atrophy. Given the ZIKV RNA shedding in the tears of infected animals, the present study shows the sequelae of direct intraocular inoculation of ZIKV that may occur during an intravitreal injection or intraocular surgery, highlighting the importance of avoiding the aforementioned clinical practices in ZIKV-infected patients.

Another major finding of our study is that type I IFN-induced ISG15 plays a protective role in the retina against ZIKV

infections. While generally considered an antiviral gene, ISG15 has been shown to influence viral replication in both a positive and a negative manner (43). Our data showed a marked increase in the expression of ISG15 in ZIKV-challenged retinal cells and mouse retinas. Our study revealed that ZIKV induced severe chorioretinitis in ISG15 $5^{-/}$mice and that the retinas of these mice had higher ZIKV RNA copies, indicating a higher viral burden. This suggests that ISG15 plays a role in inhibiting ZIKV replication in the eye. Moreover, the mRNA levels of inflammatory cytokines Tnfa and Il1b and chemokines Ccl5 and Cxcl10 were similar in WT and ISG15 ${ }^{-/}$mice, while the mRNA levels of IFNs (Ifnb and Ifng) and IFN-stimulated antiviral genes (Isg15, Oas, and $M x 1$ ) were significantly lower in ISG15 $15^{-/}$mice, suggesting that ISG15 plays a role in the positive loop of regulation of IFN signaling. Whether ISG15 functions as an ISGylation protein, as a free intracellular antiviral factor, and/or as an extracellular antiviral mediator remains to be determined.

In summary (Figure 8), our study demonstrated that retinal cells (RPE and HRvEC) are permissive to ZIKV replication and express receptors for its entry. The increased ZIKV replication and severe retinal lesions in ISG15 $5^{-/}$mice indicate an essential role of ISG15 in providing retinal defense against ZIKV infection. Impor- 


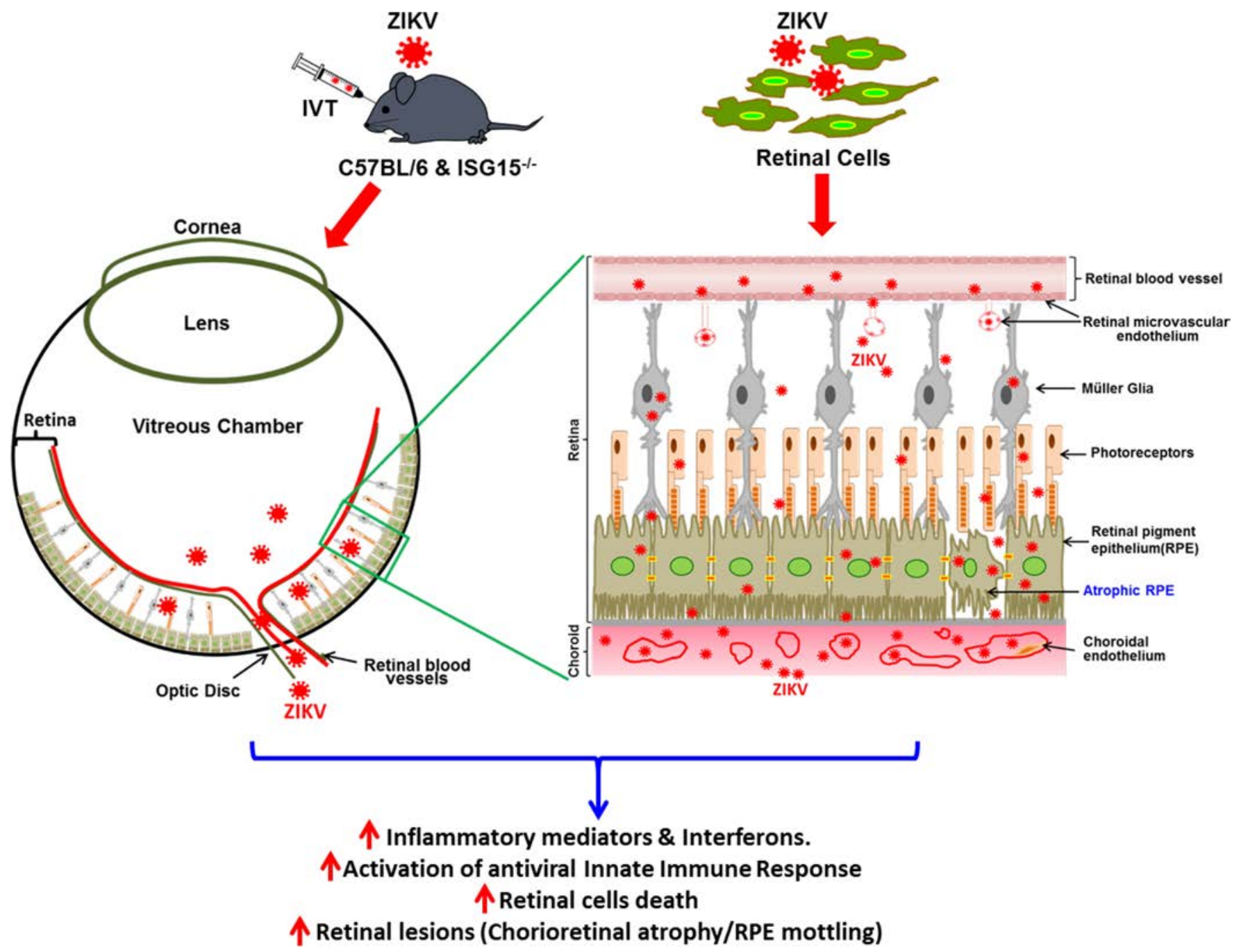

Figure 8. Schematic presentation of ZIKV pathogenesis in the eye. Intravitreal injections of ZIKV cause chorioretinal atrophy and RPE mottling in mice. ZIKV infects retinal cells lining the blood-retinal barrier, induces cell death, and initiates antiviral innate immune response.

tantly, our mouse model in which direct ZIKV inoculation in the eye results in retinal lesions can be used to investigate the mechanistic basis of ZIKV-induced ocular abnormalities and to screen new classes of therapeutics to mitigate or prevent ocular manifestations of ZIKV infection. Further studies are in progress to assess the extent of ZIKV-induced chorioretinal atrophy and the consequences this atrophy on visual outcomes in addition to studies to define molecular signatures of ZIKV infection using systems biology approaches.

\section{Methods}

Cells and culture conditions. Human Pr. RPE cells were purchased from Lonza and cultured in RtEGM Bulletkit media (Lonza). Immortal human RPE cells, ARPE-19 (ATCC CRL-2302), were grown in DMEMF12 media supplemented with 10\% FBS, $10 \mu \mathrm{g} / \mathrm{ml}$ L-glutamine, and 1\% Penicillin and Streptomycin (Invitrogen). Primary HRvEC (ACBRI 181) were maintained in M131 media supplemented with 5\% FBS, 1\% Penicillin and Streptomycin solution, and Microvascular Growth supplement (Gibco, Thermo Scientific). Macaca primary CEC (RF/6A) were grown in EMEM supplemented with 10\% FBS, $10 \mu \mathrm{g} / \mathrm{ml} \mathrm{L-glu-}$ tamine, and 1\% Penicillin and Streptomycin solution. Both HRvEC and CEC were a gift from Xiaoxi Qiao (Henry Ford Hospital, Detroit, Michigan, USA). Immortal human retinal Müller glia cells (MIOM1) $(30,61)$, provided by Astrid Limb (Moorfields Eye Hospital, London, United Kingdom), were grown in complete DMEM media supplemented with $10 \% \mathrm{FBS}, 10 \mu \mathrm{g} / \mathrm{ml} \mathrm{L-glutamine,} \mathrm{and} 1 \%$ Penicillin and Streptomycin solution. The mouse photoreceptor cell line, $661 \mathrm{~W}(31,62,63)$, provided by Muayyad A1Ubaidi (University of Oklahoma Health Sciences Center, Oklahoma City, Oklahoma, USA), was grown 
in DMEM supplemented with 10\% FBS, $10 \mu \mathrm{g} / \mathrm{ml}$ L-glutamine, 1\% Penicillin and Streptomycin, $40 \mu \mathrm{g} / 1$ hydrocortisone, $40 \mu \mathrm{g} / 1$ progesterone, $32 \mathrm{mg} / 1$ putrescine, and $40 \mu \mathrm{l} / 1 \beta$-mercaptoethanol.

Mice. Both male and female mice, at 4 to 6 weeks of age, were used in all experiments. C57BL/6 (WT) mice were purchased from Jackson Laboratory. ISG15 ${ }^{-/-}$(B6 background) mice were provided by Fu-Shin Yu (Wayne State University, Detroit, Michigan, USA) and bred in-house. Animals were housed in a restricted-access DLAR facility, maintained on a 12-hour light/dark cycle, and fed LabDiet rodent chow (LabDiet Pico lab Laboratory) and water ad libitum.

Virus strain and infection. ZIKV strains PRVABC59 (NR-50240) originally isolated from human blood in Puerto Rico in December 2015, was obtained through BEI Resources, National Institute of Allergy and Infectious Diseases, NIH. ZIKV was propagated in ATCC CCL-81 Vero cell line, and titers were determined by standard $\mathrm{TCID}_{50}$ and plaque assays. All cultured cells were infected with ZIKV at a MOI of 1 unless specified otherwise. For AXL inhibition studies, Pr. RPE cells were treated with a selective AXL inhibitor, R428 $(1 \mu \mathrm{M})$ (APExBio), for 2 hours prior to ZIKV challenge, and DMSO-treated cells along with ZIKV served as vehicle control. For in vivo studies, mice were injected with ZIKV $\left(\sim 1 \times 10^{4} \mathrm{PFU} /\right.$ eye $)$ intravitreally in the right eye. The contralateral eyes were injected with vehicle and served as mock control. At the desired time points after infection, funduscopic examination was performed using Micron III (Phoenix Research Lab). The animals were then euthanized, and enucleated eyes were subjected to viral burden determination, inflammatory cytokine/chemokine assays, and histology as described in the following sections.

Immunofluorescence staining and Western blotting. For immunostaining procedures, all cells were cultured in a 4-well chamber slide (Fisher Scientific) and infected with ZIKV at a MOI of 1. At 24, 48, and 72 hours after infection, ZIKV-infected and mock-treated cells were fixed overnight with $4 \%$ paraformaldehyde in PBS at $4^{\circ} \mathrm{C}$. For in vivo samples, 5 - $\mu$ m-thick retinal cryosections were fixed in $4 \%$ paraformaldehyde, as stated above. After washing, the cells/retinal sections were permeabilized and blocked with $1 \%(\mathrm{w} / \mathrm{v})$ BSA, 10\% (v/v) goat serum, and $0.4 \%$ Triton X-100 made in PBS for 1 hour at room temperature. Cells/retinal sections were incubated with primary mouse monoclonal antibody 4G2 (1:100) (Millipore, catalog MAB10216), cleaved caspase-3 (1:100 dilution, Cell Signaling, catalog 9664S), AXL (1:100 dilution, Cell Signaling, catalog 8661S), or ISG15 (1:100, Cell Signaling, catalog 2758S) overnight at $4^{\circ} \mathrm{C}$. Following removal of the primary antibody, the cells/retinal sections were washed extensively with PBS and incubated for 1 hour with anti-mouse/rabbit FITC/Alexa Fluor 594-conjugated secondary antibody $(1: 200)$ at room temperature. Finally, the cells/retinal sections were extensively washed with PBS, and the slides were mounted in Vectashield anti-fade mounting medium (Vector Laboratories) and visualized using an Eclipse 90i fluorescence microscope (Nikon). The Western blotting for AXL (Cell Signaling; catalog 8661S), TLR3 (Cell Signaling; catalog 6961S), HSP90 (Cell Signaling; catalog 4874S), and $\beta$-actin (Sigma-Aldrich; catalog A2228) was performed using ZIKV-infected and control cell lysates as described earlier $(31,32)$.

TUNEL. ZIKV induced cell death was assessed by TUNEL staining. Cells were grown and challenged with ZIKV in a 4-well chamber slide (Fisher Scientific) for the indicated time points. TUNEL staining was performed using the ApopTag Fluorescein In Situ Apoptosis Detection Kit as per the manufacturer's instructions (Millipore). The TUNEL-stained cells were visualized using an Eclipse 90i fluorescence microscope (Nikon).

Cell RNA extraction and $q R T-P C R$. Total RNA was extracted from ZIKV-infected cells and mouse retinas using the RNeasy Mini kit (Qiagen), as per the manufacturer's instructions. cDNA was synthesized using $1 \mu \mathrm{g}$ of total RNA using a Maxima first-strand cDNA synthesis kit, as per the manufacturer's instructions (Thermo Scientific). The cDNA was amplified using gene-specific PCR primers. qRT-PCR was conducted in a StepOnePlus Real-Time PCR system (Applied Biosystems) using TaqMan probes against various inflammatory cytokines/chemokines (TNFA, IL1B, IL6, CXCL10, and CCL5), IFNs (IFN1A, IFNB2, and IFNG), TLR3 (TLR3) and SYBR Green-based primers against receptors tyrosine kinases TYRO3, $A X L$, and MERTK (TAM), RIGI, and MDA5 as well as ISGs, such as ISG15, OAS2, and MX1. All primers and TaqMan probes (Prime Time Mini qPCR Assay) were purchased from Integrated DNA Technologies. The quantification of gene expression was determined via the comparative $\Delta \Delta \mathrm{CT}$ method. Gene expression in the test samples was normalized to the endogenous reference GAPDH level and were reported as fold change relative to $G A P D H$ gene expression.

Viral RNA isolation and $q R T-P C R$ for viral quantification. Viral RNA was isolated from cell culture supernatant and whole-eye lysates at the desired time points after infection using a QIAamp viral RNA mini kit (Qiagen), as per the manufacturer's instructions. Viral RNA transcripts were quantified using a TaqMan 
probe-based qRT-PCR by plotting a standard curve produced using serial 10-fold dilutions of ZIKV RNA as described previously $(57,64,65)$. qRT-PCR was performed in a 96-well plate on a StepOnePlus RealTime PCR system (Applied Biosystems).

Statistics. All data are expressed as the mean \pm SEM unless indicated otherwise. Statistical differences between experimental groups were determined using unpaired 2-tailed Student's $t$ test and 1-way ANOVA. All statistical analyses were performed using GraphPad Prism 7.02 (GraphPad Software). A value of $P<0.05$ was considered statistically significant. All experiments were performed at least 3 times unless indicated otherwise.

Study approval. Mice were treated in compliance with the Association for Research in Vision and Ophthalmology Statement for the Use of Animals in Ophthalmic and Vision Research. All procedures were approved by the Institutional Animal Care and Use Committee of Wayne State University under protocol A 16-06-102.

\section{Author contributions}

AK conceived the study. PKS and AK designed the study, performed experiments, analyzed the data, and wrote the manuscript. JMG assisted in animal experiments. MK assisted in manuscript writing, editing, and

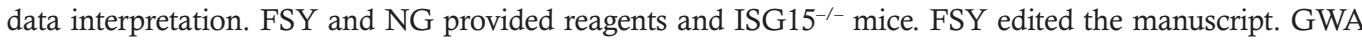
and JB performed clinical interpretations of fundus images and edited the manuscript. MSJ provided financial support and other research infrastructure. All authors read and approved the final version of the manuscript.

\section{Acknowledgments}

This study was supported by an unrestricted grant from Research to Prevent Blindness (RPB) to Kresge Eye Institute. The microscopy, imaging, and cytometry resource core is supported, in part, by an NIH center grant P30EY004068 (PI: Linda D. Hazlett, Department of Anatomy and Cell Biology, Wayne State University). We sincerely thank Rajiv Shah (Department of Ophthalmology, Wake Forest University) and Mircea Coca and Wendewessen Amde (Chicagoland Eye and Retina Foundation) for unbiased grading of fundus images. The authors are grateful to Kathy Spindler (University of Michigan, Ann Arbor, Michigan, USA) for helping us with plaque assays and Bruce G. Rottmann for critical editing of the manuscript.

Address correspondence to: Ashok Kumar, Kresge Eye Institute/Department of Ophthalmology, Wayne State University School of Medicine, 4717 St. Antoine, Detroit, Michigan 48201, USA. Phone: 313.577.6213; E-mail: akuma@med.wayne.edu.

1. Jamil Z, Waheed Y, Durrani TZ. Zika virus, a pathway to new challenges. Asian Pac J Trop Med. 2016;9(7):626-629.

2. Ribeiro LS, Marques RE, Jesus AM, Almeida RP, Teixeira MM. Zika crisis in Brazil: challenges in research and development. Curr Opin Virol. 2016;18:76-81.

3. Johansson MA, Mier-y-Teran-Romero L, Reefhuis J, Gilboa SM, Hills SL. Zika and the risk of microcephaly. N Engl J Med. 2016;375(1):1-4.

4. Mysorekar IU, Diamond MS. Modeling Zika virus infection in pregnancy. N Engl J Med. 2016;375(5):481-484.

5. Panchaud A, Stojanov M, Ammerdorffer A, Vouga M, Baud D. Emerging role of Zika virus in adverse fetal and neonatal outcomes. Clin Microbiol Rev. 2016;29(3):659-694.

6. Araujo AQ, Silva MT, Araujo AP. Zika virus-associated neurological disorders: a review. Brain. 2016;139(pt 8):2122-2130

7. Abbasi AU. Zika virus infection; vertical transmission and foetal congenital anomalies. J Ayub Med Coll Abbottabad. 2016;28(1):1-2.

8. Miranda HA, Costa MC, Frazão MA, Simão N, Franchischini S, Moshfeghi DM. Expanded spectrum of congenital ocular findings in microcephaly with presumed Zika infection. Ophthalmology. 2016;123(8):1788-1794.

9. de Paula Freitas B, et al. Ocular findings in infants with microcephaly associated with presumed Zika virus congenital infection in Salvador, Brazil. JAMA Ophthalmol. 2016;134(5):529-535.

10. Ventura CV, Maia M, Bravo-Filho V, Gois AL, Belfort R Jr. Zika virus in Brazil and macular atrophy in a child with microcephaly. Lancet. 2016;387(10015):228.

11. Ventura CV, Maia M, Dias N, Ventura LO, Belfort R. Zika: neurological and ocular findings in infant without microcephaly. Lancet. 2016;387(10037):2502.

12. Ventura CV, et al. Risk factors associated with the ophthalmoscopic findings identified in infants with presumed Zika virus congenital infection. JAMA Ophthalmol. 2016;134(8):912-918.

13. Jampol LM, Goldstein DA. ZIka virus infection and the eye. JAMA Ophthalmol. 2016;134(5):535-536.

14. Wright WF, Pritt BS. Update: The diagnosis and management of dengue virus infection in North America. Diagn Microbiol Infect Dis. 2012;73(3):215-220.

15. McKusick VA, Stauffer M, Knox DL, Clark DB. Chorioretinopathy with hereditary microcephaly. Arch Ophthalmol. 1966;75(5):597-600.

16. Atchaneeyasakul LO, Linck L, Weleber RG. Microcephaly with chorioretinal degeneration. Ophthalmic Genet. 1998;19(1):39-48.

17. Trzupek KM, Falk RE, Demer JL, Weleber RG. Microcephaly with chorioretinopathy in a brother-sister pair: evidence for germ 
line mosaicism and further delineation of the ocular phenotype. Am J Med Genet A. 2007;143A(11):1218-1222.

18. Moshfeghi DM, de Miranda H, Ii, Costa M. Zika virus, microcephaly, and ocular findings. JAMA Ophthalmol. $2016 ; 134(8): 945$.

19. Mittal A, Mittal S, Bharati MJ, Ramakrishnan R, Saravanan S, Sathe PS. Optic neuritis associated with chikungunya virus infection in South India. Arch Ophthalmol. 2007;125(10):1381-1386.

20. Mahendradas P, Avadhani K, Shetty R. Chikungunya and the eye: a review. J Ophthalmic Inflamm Infect. 2013;3(1):35.

21. Hayek S, Rousseau A, Bouthry E, Prat CM, Labetoulle M. Chikungunya virus infection and bilateral stromal keratouveitis. JAMA Ophthalmol. 2015;133(7):849-850.

22. Yip VC, Sanjay S, Koh YT. Ophthalmic complications of dengue fever: a systematic review. Ophthalmol Ther. 2012;1(1):2.

23. Li M, Zhang X, Ji Y, Ye B, Wen F. Acute macular neuroretinopathy in Dengue fever: short-term prospectively followed up case series. JAMA Ophthalmol. 2015;133(11):1329-1333.

24. Ram J, Kumar A. Intraocular and orbital hemorrhage in a patient with dengue fever during cataract surgery. JAMA Ophthalmol. 2013;131(10):1313.

25. Bains HS, Jampol LM, Caughron MC, Parnell JR. Vitritis and chorioretinitis in a patient with West Nile virus infection. Arch Ophthalmol. 2003;121(2):205-207.

26. Anninger W, Lubow M. Visual loss with West Nile virus infection: a wider spectrum of a "new" disease. Clin Infect Dis. 2004;38(7):e55-e56.

27. Miner JJ, et al. Zika virus infection in mice causes panuveitis with shedding of virus in tears. Cell Rep. 2016;16(12):3208-3218

28. Singh PK, Shiha MJ, Kumar A. Antibacterial responses of retinal Müller glia: production of antimicrobial peptides, oxidative burst and phagocytosis. J Neuroinflammation. 2014;11:33.

29. Kumar A, Shamsuddin N. Retinal Muller glia initiate innate response to infectious stimuli via toll-like receptor signaling. $P L o S$ One. 2012;7(1):e29830.

30. Shamsuddin N, Kumar A. TLR2 mediates the innate response of retinal Muller glia to Staphylococcus aureus. J Immunol. 2011;186(12):7089-7097.

31. Singh PK, Kumar A. Retinal photoreceptor expresses toll-like receptors (TLRs) and elicits innate responses following TLR ligand and bacterial challenge. PLoS One. 2015;10(3):e0119541.

32. Singh PK, Kumar A. Mitochondria mediates caspase-dependent and independent retinal cell death in Staphylococcus aureus endophthalmitis. Cell Death Discov. 2016;2:16034.

33. Talreja D, Singh PK, Kumar A. In Vivo Role of TLR2 and MyD88 Signaling in eliciting innate immune responses in Staphylococcal endophthalmitis. Invest Ophthalmol Vis Sci. 2015;56(3):1719-1732.

34. Talreja D, Kaye KS, Yu FS, Walia SK, Kumar A. Pathogenicity of ocular isolates of Acinetobacter baumannii in a mouse model of bacterial endophthalmitis. Invest Ophthalmol Vis Sci. 2014;55(4):2392-2402.

35. Tabata T, et al. Zika virus targets different primary human placental cells, suggesting two routes for vertical transmission. Cell Host Microbe. 2016;20(2):155-166.

36. Hamel R, et al. Biology of Zika virus infection in human skin cells. J Virol. 2015;89(17):8880-8896.

37. Nowakowski TJ, Pollen AA, Di Lullo E, Sandoval-Espinosa C, Bershteyn M, Kriegstein AR. Expression analysis highlights AXL as a candidate Zika virus entry receptor in neural stem cells. Cell Stem Cell. 2016;18(5):591-596.

38. Kumar A, Singh CN, Glybina IV, Mahmoud TH, Yu FS. Toll-like receptor 2 ligand-induced protection against bacterial endophthalmitis. J Infect Dis. 2010;201(2):255-263.

39. Kochan T, Singla A, Tosi J, Kumar A. Toll-like receptor 2 ligand pretreatment attenuates retinal microglial inflammatory response but enhances phagocytic activity toward Staphylococcus aureus. Infect Immun. 2012;80(6):2076-2088.

40. Pandey RK, Yu FS, Kumar A. Targeting toll-like receptor signaling as a novel approach to prevent ocular infectious diseases. Indian J Med Res. 2013;138(5):609-619.

41. Kumar A, Pandey RK, Miller LJ, Singh PK, Kanwar M. Muller glia in retinal innate immunity: a perspective on their roles in endophthalmitis. Crit Rev Immunol. 2013;33(2):119-135.

42. Morales DJ, et al. Novel mode of ISG15-mediated protection against influenza A virus and Sendai virus in mice. $J$ Virol. 2015;89(1):337-349.

43. Morales DJ, Lenschow DJ. The antiviral activities of ISG15. J Mol Biol. 2013;425(24):4995-5008.

44. Vogel G. INFECTIOUS DISEASE. Experts fear Zika's effects may be even worse than thought. Science. 2016;352(6292):1375-1376.

45. Furtado JM, Espósito DL, Klein TM, Teixeira-Pinto T, da Fonseca BA. Uveitis associated with Zika virus infection. $N$ Engl $J$ Med. 2016;375(4):394-396.

46. Parke DW, Almeida DR, Albini TA, Ventura CV, Berrocal AM, Mittra RA. Serologically confirmed Zika-related unilateral acute maculopathy in an adult. Ophthalmology. 2016;123(11):2432-2433.

47. Constable PA, Lawrenson JG. Glial cell factors and the outer blood retinal barrier. Ophthalmic Physiol Opt. 2009;29(5):557-564.

48. Coburn PS, et al. Bloodstream-to-eye infections are facilitated by outer blood-retinal barrier dysfunction. PLoS One. 2016;11(5):e0154560.

49. Kumar A, Kumar A. Role of Staphylococcus aureus virulence factors in inducing inflammation and vascular permeability in a mouse model of bacterial endophthalmitis. PLoS One. 2015;10(6):e0128423.

50. Dahlmann F, et al. Analysis of Ebola virus entry into macrophages. J Infect Dis. 2015;212(supp1 2):S247-S257.

51. Miner JJ, et al. The TAM receptor Mertk protects against neuroinvasive viral infection by maintaining blood-brain barrier integrity. Nat Med. 2015;21(12):1464-1472.

52. Meertens L, et al. The TIM and TAM families of phosphatidylserine receptors mediate dengue virus entry. Cell Host Microbe. 2012;12(4):544-557.

53. Nowakowski TJ, Pollen AA, Di Lullo E, Sandoval-Espinosa C, Bershteyn M, Kriegstein AR. Expression analysis highlights AXL as a candidate Zika virus entry receptor in neural stem cells. Cell Stem Cell. 2016;18(5):591-596.

54. Liu S, DeLalio LJ, Isakson BE, Wang TT. AXL-mediated productive infection of human endothelial cells by Zika virus. Circ Res. 2016;119(11):1183-1189.

55. Suthar MS, Aguirre S, Fernandez-Sesma A. Innate immune sensing of flaviviruses. PLoS Pathog. 2013 ;9(9):e1003541. 
56. Liang Z, et al. Activation of Toll-like receptor 3 impairs the dengue virus serotype 2 replication through induction of IFN- $\beta$ in cultured hepatoma cells. PLoS One. 2011;6(8):e23346.

57. Hamel R, et al. Biology of Zika virus infection in human skin cells. J Virol. 2015;89(17):8880-8896.

58. Dai J, Pan W, Wang P. ISG15 facilitates cellular antiviral response to dengue and West Nile virus infection in vitro. Virol J. 2011;8:468.

59. Hishiki T, et al. Interferon-mediated ISG15 conjugation restricts dengue virus 2 replication. Biochem Biophys Res Commun. 2014;448(1):95-100

60. McCarthy M. Severe eye damage in infants with microcephaly is presumed to be due to Zika virus. $B M J$. 2016;352:i855

61. Limb GA, Salt TE, Munro PM, Moss SE, Khaw PT. In vitro characterization of a spontaneously immortalized human Müller cell line (MIO-M1). Invest Ophthalmol Vis Sci. 2002;43(3):864-869.

62. Tan E, Ding XQ, Saadi A, Agarwal N, Naash MI, Al-Ubaidi MR. Expression of cone-photoreceptor-specific antigens in a cell line derived from retinal tumors in transgenic mice. Invest Ophthalmol Vis Sci. 2004;45(3):764-768

63. al-Ubaidi MR, et al. Bilateral retinal and brain tumors in transgenic mice expressing simian virus 40 large $\mathrm{T}$ antigen under control of the human interphotoreceptor retinoid-binding protein promoter. J Cell Biol. 1992;119(6):1681-1687.

64. Faye O, Faye O, Diallo D, Diallo M, Weidmann M, Sall AA. Quantitative real-time PCR detection of Zika virus and evaluation with field-caught mosquitoes. Virol J. 2013;10:311.

65. Johnson BW, Chambers TV, Crabtree MB, Guirakhoo F, Monath TP, Miller BR. Analysis of the replication kinetics of the ChimeriVax-DEN 1, 2, 3, 4 tetravalent virus mixture in Aedes aegypti by real-time reverse transcriptase-polymerase chain reaction. Am J Trop Med Hyg. 2004;70(1):89-97. 